\title{
Squamous Cell Carcinoma of the Neck of Unknown Origin -Report of 4 Cases-
}

\author{
KAZUTO NAGATA, SHIGEJIRO KURITA AND SHIGENOBU MIHASHI \\ Department of Otolaryngology, Kurume Univeristy School of Medicine, \\ Kurume, 830 Japan
}

Received for publication April 10, 1982

\begin{abstract}
Summary: Four cases of cervical lymphnode metastasis of squamous cell carcinoma of unknown origin were reported. Three of these 4 patients were treated with radical neck dissection, and one patient was treated with removal of the tumor. Three patients died within 1 year from the initial treatment and 1 patient died from a neck recurrence 3 years and 5 months after the initial treatment.
\end{abstract}

Key words: carcinoma - cervical metastasis - occult carcinoma

\section{Introduction}

Head and neck surgeons occasionally encounter cervical metastatic nodes of unknown origin. During the period from 1971 to 1980 , we treated 4 cases of squamous cell carcinoma of the neck of unknown origin in Kurnme University Hospital. This paper describes report of these cases.

\section{Case reports}

Case 1: S.S.

A 60-year-old female noticed a tumor formation in her right cervical region in January, 1972. The tumor gradually increased in size, and she subsequently visited a private clinic 2 months later. A biopsy was done on March 24, 1972 and was diagnosed as a moderately differentiated squamous cell carcinoma. She was referred to our hospital on April 26, 1972. A $5 \mathrm{~cm}$ $\times 3 \mathrm{~cm}$ elastic hard tumor was palpated in the right supraclavicular region. She was admitted in our hospital on May 6, 1972. $\mathrm{X}$-ray examinations, lymphography, scin- tigraphy and angiography revealed no evidence of malignant primary lesions in the head and neck. Examinations revealed the stomach, breast and lung did not present any primary tumor. Also chemical examinations of the blood serum revealed no abnormalities.

On May 15, 1972, radical neck dissection was performed on the right side. The spinal accessory nerve had to be removed because of tumor invasion. Several enlarged lymphnodes were found in the lower deep cervical region. Four of 10 lymphnodes were histologically proven to be moderately differentiated squamous cell carcinoma. In the largest lymphnode $(5 \mathrm{~cm} \times 3 \mathrm{~cm} \times 1 \mathrm{~cm})$, extracapsular invasion was found. Intravenous Bleomycin, $270 \mathrm{mg}$ in total, was given to the patient from May 9 to June 13 .

She was discharged from our hospital on June 14, 1972 without any evidence of recurrent tumor. A recurrent tumor on the right neck was detected at another clinic 5 months after her discharge. She died on January 15, 1973 in that hospital. The family refused to have an autopsy completed on the deceased lady. 
Case 2: A. N.

A 69-year-old male complained of a tumor formation in his right cervical region early in October 1979. He visited a private hospital and a $2 \mathrm{~cm} \times 2 \mathrm{~cm}$ neck tumor was discovered. However, he refused any surgical treatment. Since the neck tumor had gradually increased in size, he visited our hospital on May 15, 1980. A large firm mass, $7 \mathrm{~cm} \times 7 \mathrm{~cm}$ in size, was palpated in the right cervical region. The tumor appeared to be fixed to the carotid artery and the internal jugular vein. He was admitted in our hospital on May 20, 1980. Carotid angiography showed that the inner surface of the carotid artery was smooth and regular, suggesting no tumor invasion into the inside of the artery. Careful physical and $\mathrm{X}$-ray examinations revealed no primary lesion in the head and neck. Examinations of the entire body did not detect and primary lesion.

Extirpation of the neck tumor was attempted. A capsulated large mass was seen beneath the platisma muscle. It was difficult to elevate the tumor from the carotid artery. The posterior part of the tumor appeared to have invaded the cervical vertebrae. Removal of the tumor was impossible because of deep invasion. Histological analysis revealed that the tumor was a moderately differentiated squamous cell carcinoma. He was discharged 13 days after the operation and referred to another hospital for palliative radiotherapy and chemotherapy. He died from the neck tumor on December 14, 1980. The family refused to have an autopsy completed on the deceased man.

Case 3: T. O.

A 50-year-old male noticed a swelling in the right submandibular region in April, 1970. He developed another tumor in the left submandibular region in July. The tumors had no tendency of increasing in size.

He complained of pain at swallowing in
August, 1970. Tonsillectomy was performed under the diagnosis of acute tonsillitis in our out patient clinic on September 18, 1970. The neck tumors were not treated at that time and follow up examinations were planned.

The bilateral neck tumor gradually increased in size. The right tumor measured $3.5 \mathrm{~cm} \times 4.0 \mathrm{~cm}$ and left one was $5.0 \mathrm{~cm} \times$ $6.5 \mathrm{~cm}$ in size on October, 5. He was admitted immediately to our hospital on October 7, 1970. Careful physical and X-ray examinations, revealed no evidence of any primary lesion in head and neck. Biopsy was done from the left tumor on October 8 , and confirmed a moderately differentiated squamous cell carcinoma.

On October 19, 1970, radical neck dissection was performed on the right side. During the operation, the tumor, $4.9 \mathrm{~cm}$ $\times 3.5 \mathrm{~cm}$ in size, was located in the submandibular region and was not fixed to the carotid artery. One of the middle deep cervical nodes, $1.9 \mathrm{~cm} \times 1.6 \mathrm{~cm}$ in size, was suspected to be a metastasis. They were moderately differentiated squamous cell carcinoma in histological examination. Bleomycine, $300 \mathrm{mg}$ in total, was given to the patient after the operation.

Radical neck dissection on the left side was performed on Nobember, 16. Histological examination showed no evidence of malignancy in cervical lymphnodes. He was discharged without any evidence of recurrent tumor on January 26, 1971. After the discharge, routine follow up examinations were indicated, but the patient did not come back. A recurrent tumor on the neck was noted at a private hospital 7 months later and the patient died from the recurrent neck tumor at the hospital 8 months after the initial treatment. Autopsy was not done.

Case 4: C. K.

A 62-year-old female noticed a tumor formation on the right submandibular region in June, 1972. She visited another 
clinic and was diagnosed as a sialolithiasis. The tumor was untreated for two years. The tumor gradually increased in size from May, 1974. She visited our hospital on June 14, 1974. A $5 \mathrm{~cm} \times 3 \mathrm{~cm}$ elastic hard tumor was palpated on the right submandibular region. But the tumor was not related to the submandibular gland. The patient was admitted to our hospital on July 16, 1974. Careful physical and X-ray examinations revealed no evidence of any primary malignacy in the head and neck region. Radical neck dissection on the right side was performed on July 22, 1974. The tumor was fixed to the carotid artery and the subcutanous connective tissue, however, removal of the tumor was successfully done. The tumor was diagnosed as a moderately differentiated squamous cell carcinoma. She was discharged without any evidence of recurrent tumor on August 7, 1974. A routine follow up examination once a month was planned in our outclinic.

On August 27, 1976, 2 years after the initial treatment, a firm tumor in the right submandibular region was pointed out and the patient was immediately admitted again to our hospital. Thorough examinations of the entire body detected no primary lesion. Carotid angiography presented that the inner surface of the carotid artery was smooth and regular. Extirpation of the tumor was attempted on September 2, 1976. The tumor was fixed to the bifurcation of the carotid artery, but the tumor was removed completely. The tumor was diagnosed as a moderately differentiated squamous cell carcinoma. The patient was discharged on October 6, 1976. without any recurrent tumor.

One month and a week after the discharge, she died from unknown disease in a private hospital. There were no evidence of recurrent tumor. Autopsy was not accepted.

\section{Discussion}

Cervical lymphnode metastasis may occur from malignant tumor of any organs. Taketa et al. (1972) reported 159 cervical lymphnode metastases, which were diagnosed as a squamous cell carcinoma. Of these 159 cases, $97(61 \%)$ were from the carcinoma of the head and neck region, $40(25.2 \%)$ were from the other sites and 22 (18\%) were from unknown origin. Of the 97 cases of the head and neck region, 35 were from the nasopharynx, 21 from the oral cavity, 17 from the larynx, 13 from the maxillary sinus, 6 from the salivary gland, 2 from the tonsil, 2 from the thyroid gland and 1 from the middle ear. Of the 40 cases of the other sites, 27 were from the lung, 8 from the esophagus, 2 from the stomach, 2 from the cervix of the uterus and 1 from some other site. Of the 53 biopsies from metastatic cervical nodes reported by Taketa et al. (1972), 29 were from the lymphnodes besides the supraclavicular area and 24 from the supraclauicular area. Of the former 29 cases, $24(83 \%)$ had primary cancers in the head and neck region. Of the latter 24, 19 (79\%) had the primary lesion in the organs caudal to the clavicula. From this data, head and neck surgeons should primarily examine thoroughly the head and neck region if the metastatic node is found in the regions except the supraclavicular area.

Jesse et al. (1973) reported the results of the treatment of 210 cases of cervical lymphnode metastasis, in which no primary cancer was found. Of these 210 cases, 184 were available for the study of 3 year-survival rate. Of the 184 cases, 104 were treated with surgery and the 3 year-survival rate was $57 \%$. Twenty-eight were treated with surgery combined with irradiation and the 3 year-survival rate was $47 \%$. The other 52 cases were treated by irradiation and the 3 year-survival rate was $48 \%$. 
Of our 4 cases, one was a inoperable case. The other 3 cases were treated with radical neck dissection and experienced a neck recurrence. Two of them were not treated for the recurrent tumor and died within 1 year. The other was treated for the recurrent tumor with surgery and the recurrent tumor was controlled. But this patient died from unknown disease 3 years and 5 months after the initial treatment. Radical neck dissection afforded the prolonging the life of this case. Radical neck dissection should be recommended for the initial treatment if a thorough examination of the entire body can not reveal any primary lesion.

\section{References}

Taketa, C., Ono, I., Ebihara, S., Sano, R. and Hirata, E. (1972). Survey of the occult carcinoma cases with lymphnodal metastatases to the neck from clinicopathological viewpoint. Japanese Journal of Cancer Clinics, 18, $185-190$.

Didolkar, M., Fanous, N., Elias, E. and Moore, R. (1977). Metastatic carcinoma from occult primary tumors. (A study of 254 patients.) Ann. Surg. 186, 625-630.

Jesse, R., Perez, C. and Fletcher, G. (1973). Cervical lymph node metastasis: unknown primary cancer. CANCER, 31, 854-859.

МАссомв, M. (1972). Diagnosis and treatment of metastatic cervical cancerous nodes from an unknown primary site. Am. J. Surg. $124,441-449$. 